\title{
Stability of health-related quality of life and morbidity burden from 18 months after diagnosis of prostate cancer: results of a UK-wide population-based outcome cohort
}

\author{
Samantha J. Mason ${ }^{1} \cdot$ Amy Downing ${ }^{2,3}$ (1) Sarah Wilding ${ }^{4} \cdot$ Luke Hounsome $^{5} \cdot$ Penny Wright $^{2} \cdot$ Eila Watson $^{6}$. \\ Richard Wagland ${ }^{7} \cdot$ Hugh Butcher $^{2} \cdot$ Paul Kind $^{8} \cdot$ Peter Selby $^{2,9} \cdot$ Anna Gavin $^{10} \cdot$ Adam W. Glaser $^{2,3}$
}

Received: 17 March 2021 / Accepted: 25 October 2021 / Published online: 13 December 2021

(c) The Author(s) 2021

\begin{abstract}
Objective To evaluate the dynamic nature of self-reported health-related quality of life (HRQL) and morbidity burden in men diagnosed with prostate cancer, we performed a follow-up study of the Life After Prostate Cancer Diagnosis (LAPCD) study cohort 12 months after initial survey.

Methods The LAPCD study collected information from 35,823 men across the UK who were 18-42 months post-diagnosis of prostate cancer. Men who were still alive 12 months later were resurveyed. Generic HRQL (EQ-5D-5L plus self-assessed health rating) and prostate cancer-specific outcomes (EPIC-26) were assessed. Treatment(s) received was self-reported. Previously defined clinically meaningful differences were used to evaluate changes in outcomes over time.

Results A total of 28,450 men across all disease stages completed follow-up surveys ( $85.8 \%$ response). Of the 21,700 included in this study, $89.7 \%$ reported no additional treatments since the first survey. This group experienced stable urinary and bowel outcomes, with good function for most men at both time points. On-going poor (but stable) urinary issues were associated with previous surgery. Sexual function scores remained low (mean: 26.8/100). Self-assessed health ratings were stable over time. The largest declines in HRQL and functional outcomes were experienced by men reporting their first active treatment between surveys.

Discussion The results suggest stability of HRQL and most specific morbidities by 18-42 months for men who report no further treatment in the subsequent 12 months. This is reassuring for those with good function and HRQL but re-enforces the need for early intervention and support for men who experience poor outcomes.
\end{abstract}

Keywords Prostate cancer · Patient-reported outcomes · Survivorship · Health-related quality of life $\cdot$ Health status · Functional outcomes

Anna Gavin and Adam W. Glaser are joint senior authors.

Amy Downing

a.downing@leeds.ac.uk

1 School of Human and Health Sciences, University of Huddersfield, Huddersfield, UK

2 Leeds Institute of Medical Research at St James's, University of Leeds, Leeds, UK

3 Leeds Institute for Data Analytics, University of Leeds, Level 11, Worsley Building, Leeds LS2 9NL, UK

4 School of Psychology, University of Leeds, Leeds, UK

5 Public Health England, Bristol, UK
6 Oxford Institute of Nursing, Midwifery and Allied Health Research, Oxford Brookes University, Oxford, UK

7 Faculty of Health Sciences, University of Southampton, Southampton, UK

8 Academic Unit of Health Economics, University of Leeds, Leeds, UK

9 Leeds Teaching Hospitals NHS Trust, Leeds, UK

10 Northern Ireland Cancer Registry, Queens University Belfast, Belfast, UK 


\section{Introduction}

Men are living for increasing periods with and beyond a diagnosis of prostate cancer (PCa) [1]. In light of this, focus has shifted to understanding the needs of men surviving PCa and their health-related quality of life (HRQL) in the years following diagnosis and treatment [2].

It is believed that the most severe treatment side effects occur in the first year following treatment for $\mathrm{PCa}$, with some improvement thereafter [3-6]. Studies have found that surgery has the greatest detrimental impact on urinary continence and erectile function, radiotherapy is most associated with bowel and urinary irritation problems [3-6] and androgen deprivation therapy (ADT) has a range of adverse side effects, such as sexual dysfunction, fatigue and problems with emotional wellbeing [7-9].

Many HRQL studies focus on the impact of specific primary treatments, typically in men with localised $\mathrm{PCa}$ $[3,4,7,10]$. Few studies have addressed longer-term outcomes $[7,11]$, particularly in patients treated with a range of regimens and those with advanced disease.

The UK-wide Life After Prostate Cancer Diagnosis (LAPCD) study [12] adopted an established approach to the measurement of population-level HRQL, previously used in a national population of colorectal cancer survivors [13]. Over 35,000 men 18-42 months post-diagnosis completed the first LAPCD survey, and results showed that while HRQL was generally good, a high level of sexual dysfunction was experienced across the cohort and substantial problems with hormonal function and fatigue were reported, particularly by men treated with ADT [14]. Results supported previous findings showing that surgical patients experienced the worst continence and radiotherapy patients reported more bowel issues than other treatment groups [14].

Given that the most severe side effects of PCa treatment are reported during the first year, it might be assumed that the acute consequences of initial treatment, particularly surgery and radiation, will have settled to a stable level by 18 months post-diagnosis. However, little is known about whether HRQL remains stable, improves or deteriorates in the medium to long-term. To evaluate the dynamic nature of self-reported HRQL and morbidity burden, we performed a follow-up study of the LAPCD cohort approximately 12 months after completion of the initial survey.

\section{Patients and methods}

\section{Sample}

The LAPCD study design has been detailed previously [12]. Briefly, men alive 18-42 months after a PCa diagnosis were invited to participate in the first LAPCD survey from October 2015 to November 2016. They were identified through national cancer registration systems in England, Wales and Northern Ireland. Patients from Scotland were identified through hospital activity data. Men were sent postal surveys on behalf of their NHS provider and consented by returning completed surveys. Men who completed the first survey were re-surveyed 12 months later. Up to two reminders were sent to non-responders. Before survey mail-outs and reminders, a death check was carried out to ensure that men who had recently died were not contacted. The study received ethics and governance approvals from the following organisations: Newcastle and North Tyneside 1 Research Ethics Committee (15/NE/0036), Confidentiality Advisory Group (15/ CAG/0110), NHS Scotland Public Benefit and Privacy Panel (0516-0364) and NHS Research and Development approval from Wales, Scotland and Northern Ireland.

\section{Survey content}

Survey content was the same at both time points, except for questions about how men were diagnosed, employment status at diagnosis and ethnicity, which were not included in the follow-up survey as they would not have changed. Questions were included about treatments received, generic HRQL (EQ-5D-5L $[15,16])$ and PCa specific outcomes (Expanded Prostate Cancer Index Composite-26 [EPIC-26 [17]]) along with sociodemographic information and presence of other long-term conditions (LTCs).

EQ-5D-5L records problems on five dimensions (mobility, self-care, usual activities, pain/discomfort and anxiety/ depression), plus a rating of self-assessed health (SAH) based on 'how good or bad your health is today' (valued $0-100$, where 100 represents best possible health). There are five response options for the domains ranging from no problems to extreme problems.

EPIC-26 measures function over five domains (urinary incontinence, urinary irritation and obstruction, bowel function, sexual function and vitality and hormonal function). Domain scores range from 0 to 100 , with 100 representing best possible function. Items are scored on either a four or five point scale [18].

\section{Data analysis}

Stage at diagnosis was obtained from national cancer registration records and categorised as I/II (localised), III (locally advanced) and IV (metastatic). An area-based measure of socio-economic deprivation (split into quintiles) was derived using postcode of residence [19-22]. Age (at first survey) and treatment were derived from the survey data. 
Age was grouped into $<55$ years, $55-64$ years, $65-74$ years, $75-85$ years and $\geq 85$ years.

Information on treatment(s) was taken from the questionnaire and grouped into single therapies (e.g. surgery or external beam radiotherapy [EBRT]) or combination therapies (e.g. EBRT and ADT). Analysis was restricted to men who reported receiving one of the most common single or combination treatments, as reported in earlier LAPCD work [14] (Fig. 1)and excluded those who were unsure about combination of treatments. Respondents were categorised into three groups: those who self-reported no further treatment at the time of the follow-up survey, those who reported receiving their first active treatment at follow-up (and were previously on active surveillance (AS) or watchful waiting (WW)) and those who reported receiving additional active treatment at follow-up.

For EQ-5D-5L, the proportion of respondents reporting any problem, regardless of severity, in each dimension
Fig. 1 Flow diagram of inclusions and exclusions

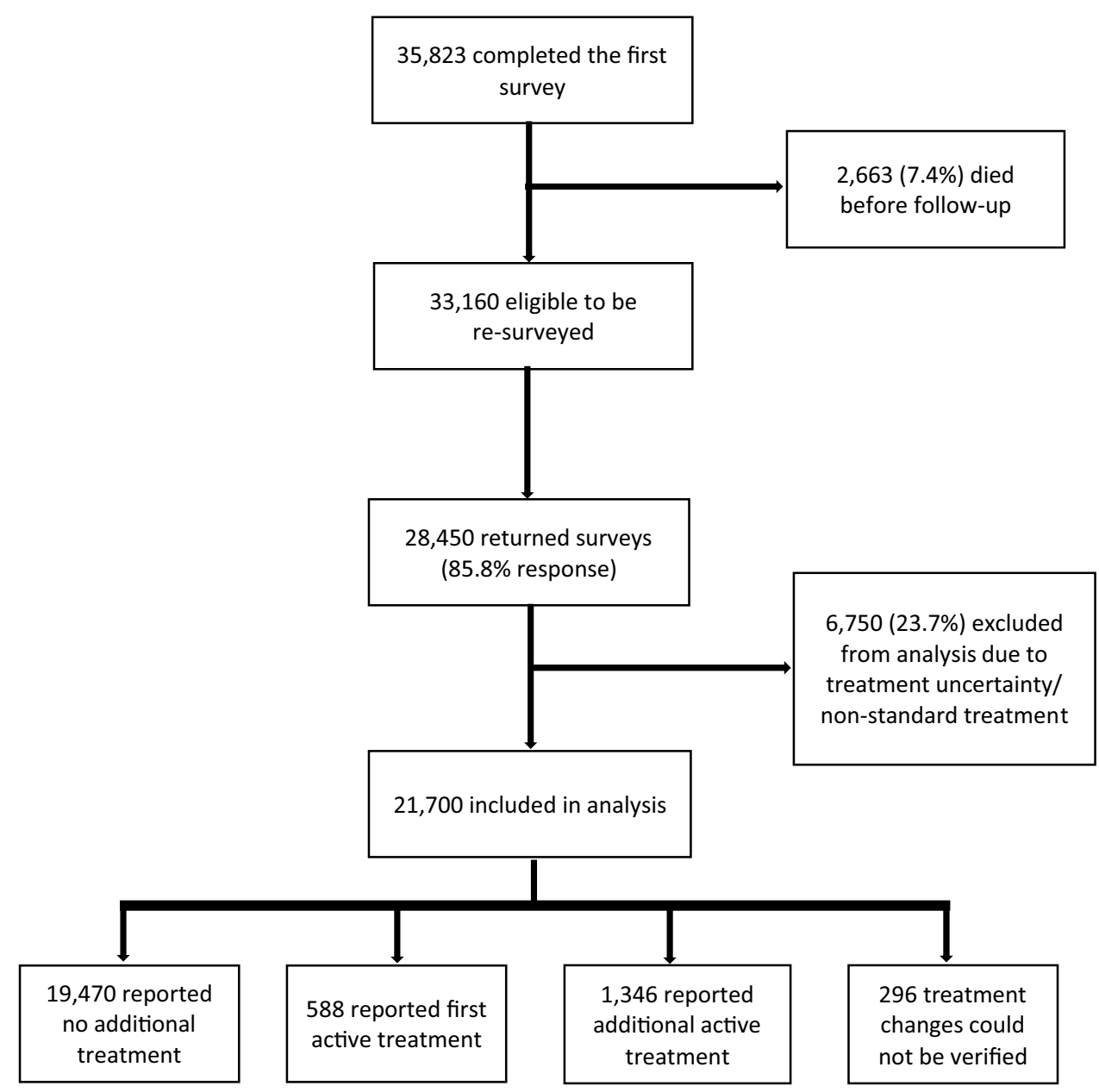

\begin{tabular}{|l|l|}
\hline \multicolumn{1}{|c|}{ Included in analysis $(\mathbf{n = 2 1 , 7 0 0 )}$} & \multicolumn{1}{c|}{ Excluded from analysis $(\mathbf{n}=\mathbf{6 , 7 5 0 )}$} \\
\hline Surveillance & High intensity focused ultrasound (HIFU) \\
\hline Surgery & Radiotherapy type unknown \\
\hline External Beam Radiotherapy (EBRT) & Follow-up unknown \\
\hline Androgen deprivation therapy (ADT) & Other non-standard combination \\
\hline Brachytherapy & No treatment \\
\hline Surgery \& EBRT (+/- ADT) & \\
\hline EBRT \& ADT & \\
\hline Systemic treatment \& EBRT (+/-ADT) & \\
\hline Systemic treatment \& ADT & \\
\hline
\end{tabular}

what treatment they received or reported a non-standard

separately and across all five dimensions was derived. Mean 
SAH ratings were calculated. To compare changes in SAH scores between the two surveys, a previously defined clinically meaningful difference (CMD) of 7 points was used [23].

Mean scores were calculated for each EPIC-26 domain. In addition, individual item responses were used to derive the proportion of respondents that reported a moderate/ big problem (or equivalent) [24]. To compare changes in EPIC-26 domain scores over time, previously defined figures representing CMDs were used [25]. In addition, men who reported poor functional outcomes at first survey (EPIC-26 domain scores of $<50$, apart from the sexual domain where scores $<10$ were used) were analysed separately. These scores were below the average domain scores and thus represented poor function.

Descriptive statistics were used to report respondent characteristics, EQ-5D-5L and EPIC-26 responses. Outcomes were analysed in relation to age, stage and type of treatment. Analyses were based on patients who provided answers to questions in both the original and follow-up surveys unless otherwise stated. Analyses were performed using Stata version 15 (Stata, College Station, TX).

\section{Results}

\section{Sample size and response rates}

Of the 35,823 men who completed the initial survey, 2663 (7.4\%) men died in the period between surveys, giving a final sample of 33,160 men eligible to complete the followup survey. Of these, 28,450 returned completed surveys (85.8\% response rate) (Fig. 1).

Men who did not complete the follow-up survey were more likely to have advanced disease (stage IV at diagnosis), be $\geq 85$ years old and have reported $\geq 4$ LTCs in the first survey.

\section{Characteristics of the study population}

Analyses were performed on 21,700 men who reported receiving one or more of the single or combination therapies detailed in Fig. 1 in both surveys. Table 1 details the characteristics of these men. Half of the cohort (49.5\%) were aged 65-74 at the first survey, and over half (56.9\%) had stage I or II disease at diagnosis, $20.3 \%$ had stage III and $9.1 \%$ had stage IV cancer.

Most men reported no additional treatments since the first survey $(19,470 / 21,700,89.7 \%)$, including $15.4 \%$ $(3039 / 19,740)$ who reported no active treatment (AS or $\mathrm{WW}$ ) at both time points. The remaining $10.3 \%$
Table 1 Patient and tumour characteristics split by treatment status at follow-up

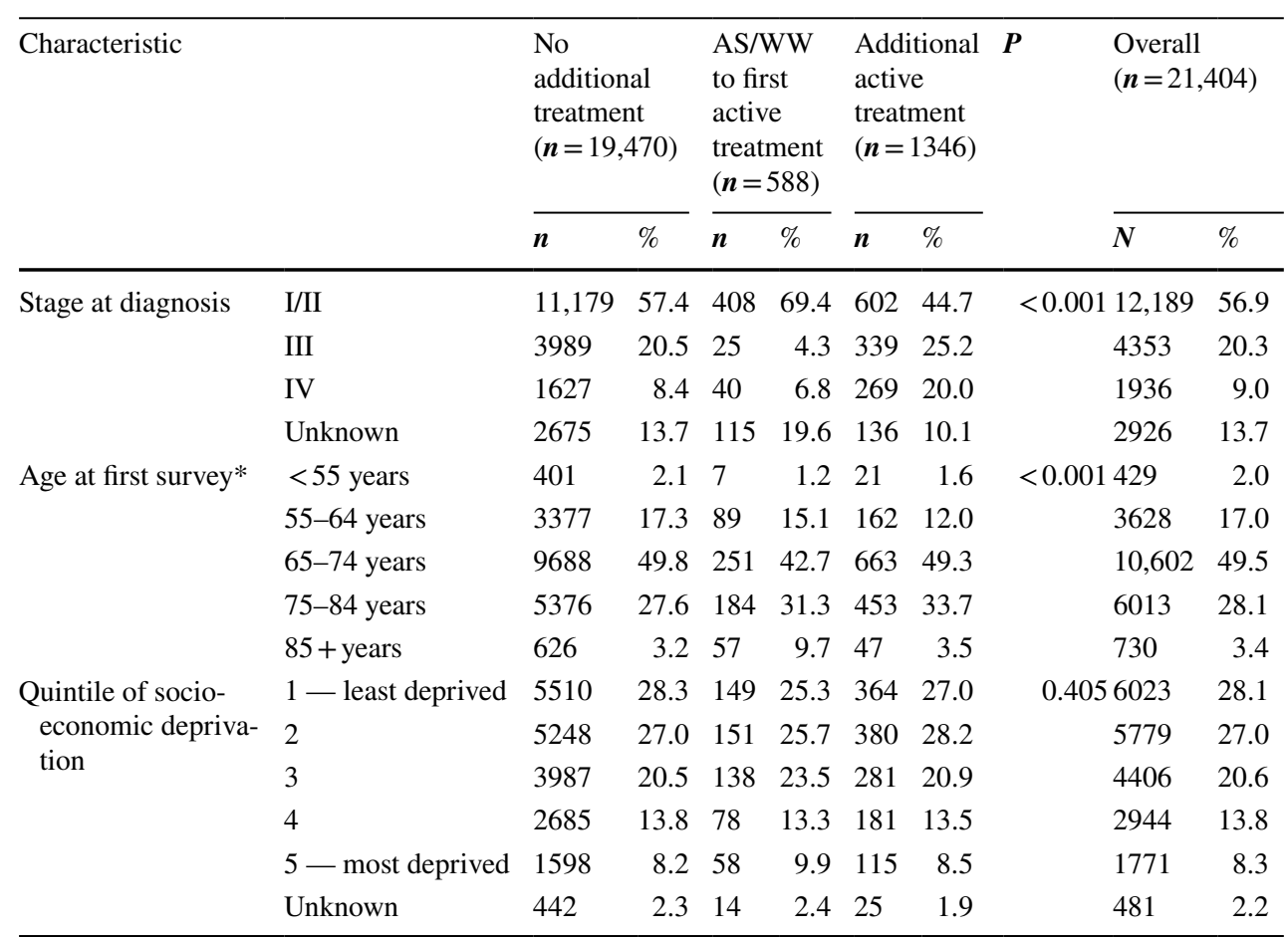

*Age was unknown for 2 men 
$(2230 / 21,700)$ reported additional treatment at follow-up. We were unable to verify the treatment changes reported by 296 respondents (Supplementary table 1). These respondents were excluded from analysis leaving 1934 men who received additional treatment (Table 1). Of these 1934 men, $588(30.4 \%)$ reported no active treatment at first survey (AS or WW) and active treatment at follow-up: the most common subsequent treatments were surgery alone $(n=234)$, ADT alone $(n=172)$ and combined EBRT and ADT $(n=78)$. A further 29\% (560/1934) of men reported the addition of ADT following initial EBRT treatment, $16.1 \%(312 / 1934)$ reported moving to systemic treatment and $12.9 \%$ (250/1934) reported additional EBRT and ADT following surgery (Supplementary table 2).

\section{Respondents who reported no additional active treatment at follow-up}

\section{Urinary and bowel function}

Mean urinary incontinence scores were high in the original (82.8/100) and follow-up surveys (81.9/100), indicating good function. Surgical treatment had the largest impact on continence, with this group reporting the lowest scores in the original and follow-up surveys (73.9/100 and 74.2/100 respectively for the surgery alone group). No CMDs in scores were observed across age, stage, or treatment groups (Table 2). Poor continence (score < 50) was reported by $10 \%$ of men $(n=1683)$ in the first survey. At follow-up, $70 \%$ of these men continued to report poor continence (mean score 28.8) (Table 3).

Overall, bowel function scores were high with no observed change at follow-up (90.1/100 in both surveys) (Table 2). Compared to other domains, a small proportion of men reported poor bowel scores $(<50)$ in the first survey (4.2\%, 678 men, mean score 37.5). Around half of these men (48\%) reported continued poor bowel function scores (mean score 35.5) at follow-up (Table 3).

\section{Vitality and hormone function}

The largest improvements at follow-up were reported in this domain, with increases in scores across all stages and age groups. Men treated with combined EBRT and ADT reported a CMD in hormone function at follow-up $(+5.3$ points, mean score 78.3) (Table 2). Fewer men indicated they had moderate/big problems with hot flushes (16\% at follow-up compared to $29 \%$ in the original survey) and changes in body weight (17\% compared to $22 \%$ in the original survey). Low hormone domain scores $(<50)$ were reported by $10 \%$ of respondents $(1669$, mean score 37.2$)$ in the first survey. At follow-up, $50.7 \%$ of this group continued to report low scores in this domain (mean score 35.1) (Table 3).

\section{Sexual function}

Mean scores for sexual function remained poor at follow-up $(+0.3$ points, mean score 26.8$)$, with scores much lower than for other domains in both the first and follow-up surveys (Table 2). In terms of perceived 'bother', similar numbers reported their (lack of) sexual function to be a moderate or big problem $(44.9 \%$ in the original survey and $44.1 \%$ at follow-up). One-third of men scored $<10 / 100$ in the first survey (34.3\%; mean score 2.8 ). Of these, $71.1 \%$ continued to report very poor sexual function at follow-up (mean score 2.3) (Table 3). Men treated with ADT reported the worst sexual function at follow-up (mean scores ranged from 12.5 for ADT alone to 21.0 for combined EBRT and ADT) (Table 2). Men treated with ADT also reported the largest proportion of 'poor/very poor' responses when asked about their ability to have an erection $(89 \%)$.

\section{Generic HROL}

There were small increases (1-3\%) in the proportion of men reporting problems at follow-up on the EQ-5D-5L dimensions, except for anxiety/depression. Overall SAH was stable over time (decreasing 0.2 points to 78.9) and across age, stage and treatment groups. Respondents diagnosed with stage IV cancer and those aged $\geq 85$ years reported more problems in all EQ-5D dimensions and worse SAH at follow-up, although these differences were not clinically meaningful (Table 4).

\section{Respondents on monitoring at first survey who then received active treatment}

\section{Urinary and bowel function}

In the group who reported surgery as their first active treatment at follow-up (alone or combined with EBRT and ADT), there was a CMD in the reporting of urinary incontinence: mean scores decreased by 11.9 points, indicating poorer function (Table 2). Worse urinary irritation and bowel function were reported by men whose first active treatment included EBRT (alone or combined with ADT) or brachytherapy. CMDs in mean urinary irritation and bowel function scores were observed at follow-up ( -6.6 points and -8.3 points, respectively).

\section{Vitality and hormone function}

Declines in hormone function were reported by men who had moved to an active treatment at follow-up. These declines were largest (around 10 points) for men who had 


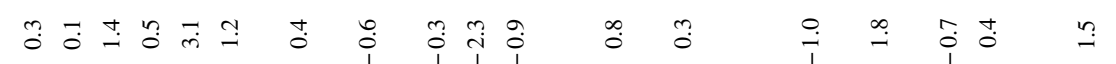

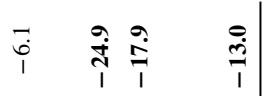

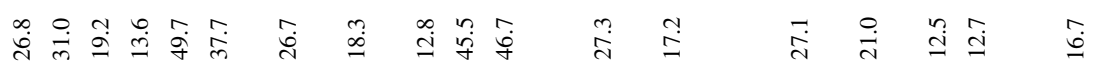

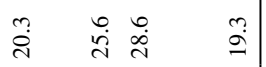

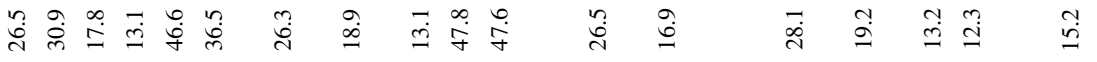

t)

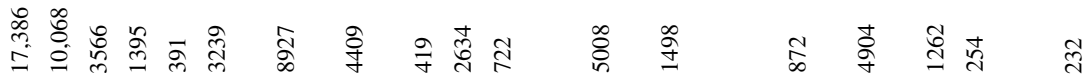

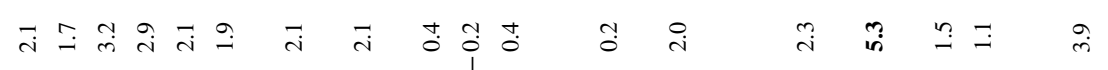

声

o.

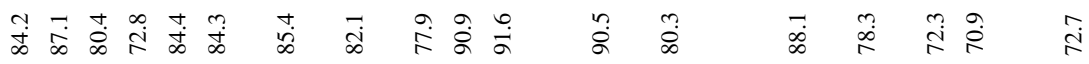

$\stackrel{\substack{\infty \\ \infty}}{\infty} \underset{\infty}{\infty}$

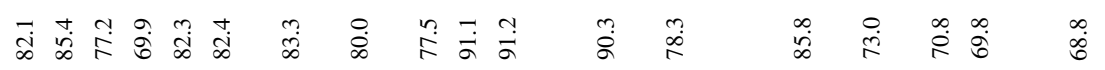

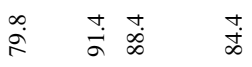

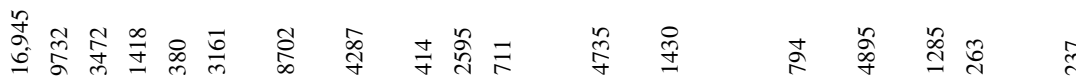

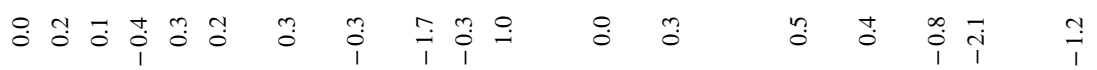

苟 2

$\stackrel{\infty}{i} \quad \stackrel{0}{\circ} \underset{0}{1}$

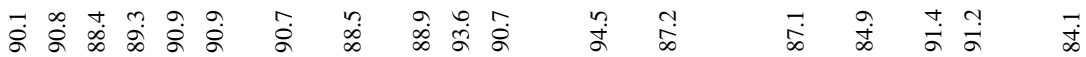

$\begin{array}{lll}\infty & \infty & 0 \\ \infty & \stackrel{2}{\infty} & + \\ \infty & +\end{array}$

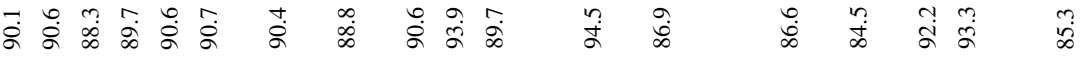

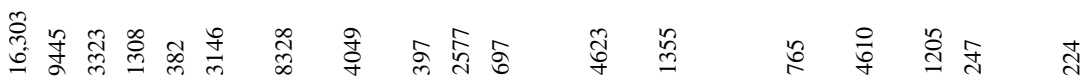

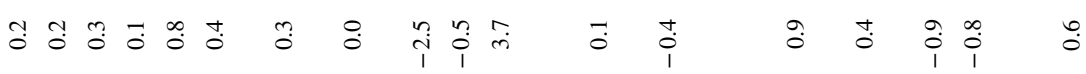

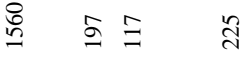

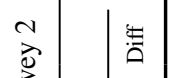

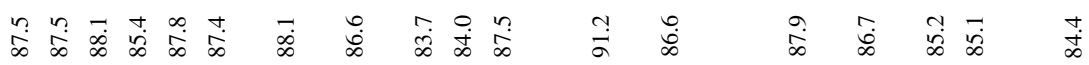

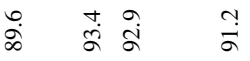

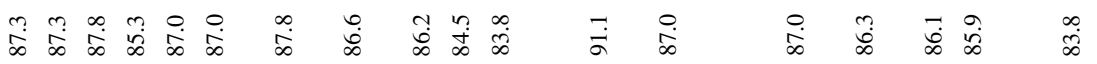

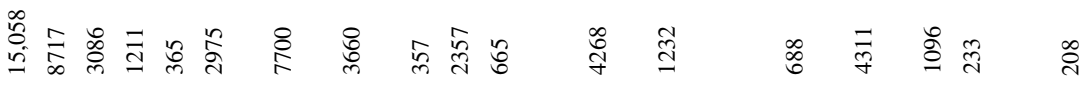

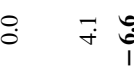

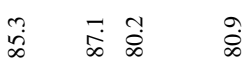

oे ố

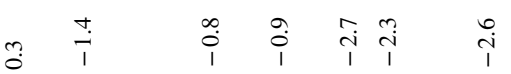

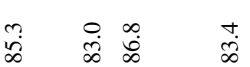

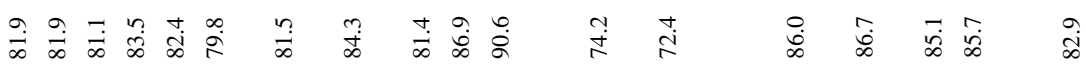

$\stackrel{\leftrightarrow}{9} \cong$ वे

(1)

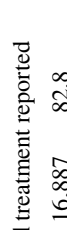

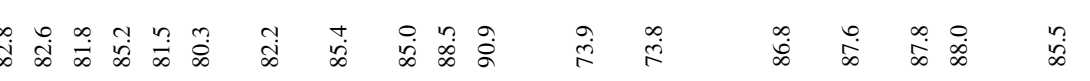

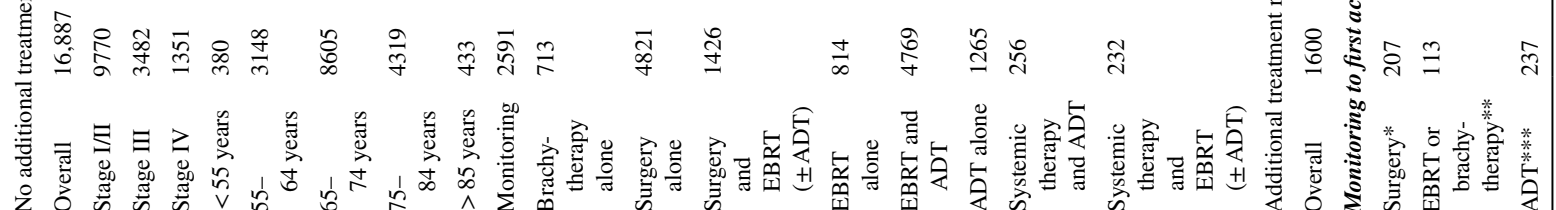

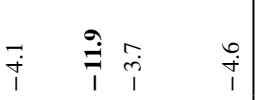




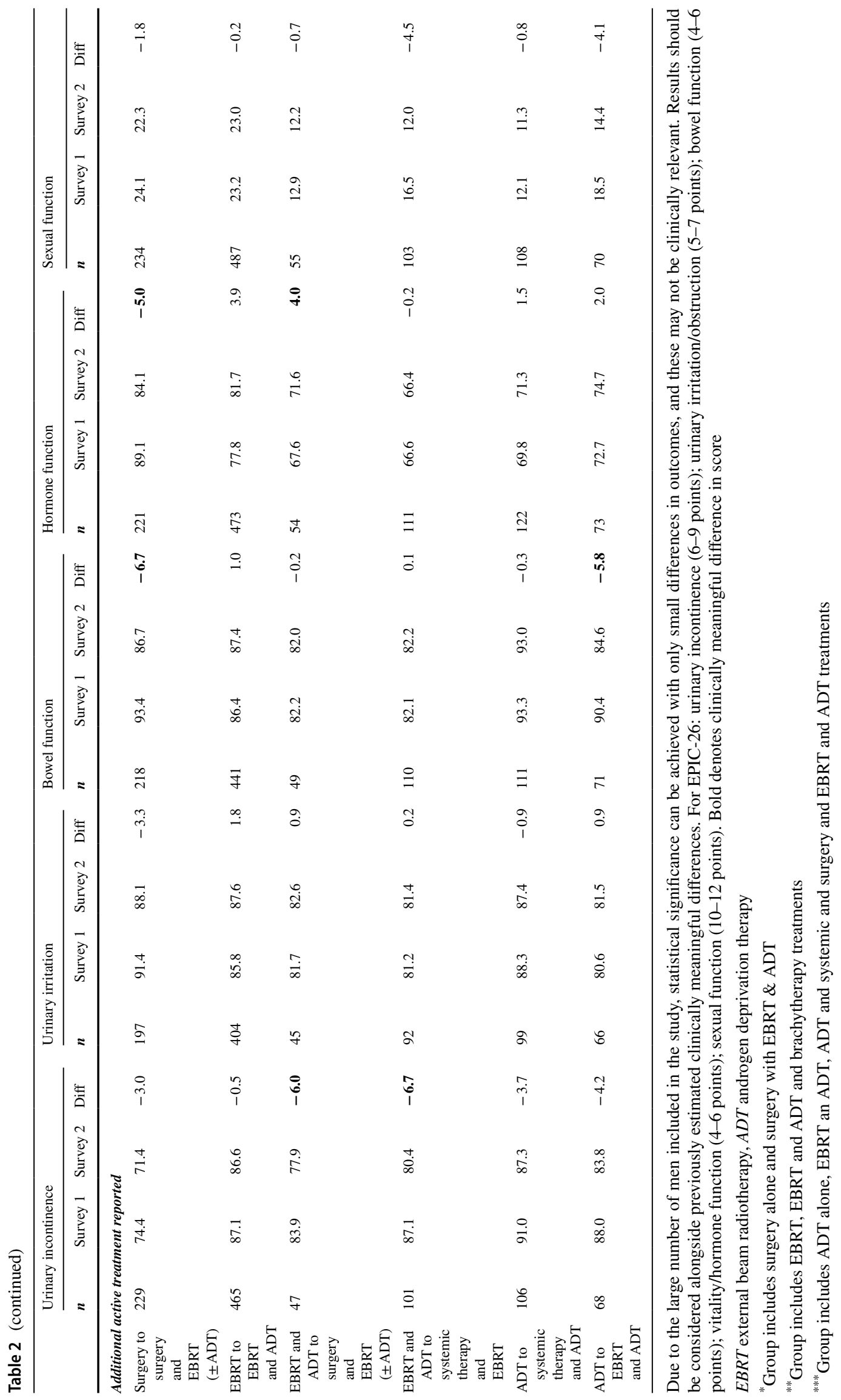


Table 3 Percentage of men reporting poor functional outcomes in survey 1 and survey 2 (in men who reported no further treatment at follow-up)

\begin{tabular}{|c|c|c|c|c|c|c|c|}
\hline & \multirow[t]{2}{*}{ Total no. men } & \multicolumn{3}{|c|}{ Poor function in survey 1} & \multicolumn{3}{|c|}{ Poor function in survey 2} \\
\hline & & $n$ & $\%^{\mathrm{a}}$ & Mean & $n$ & $\%^{\mathrm{b}}$ & Mean \\
\hline Urinary incontinence & 16,887 & 1683 & 10.0 & 32.1 & 1177 & 69.9 & 28.8 \\
\hline Urinary irritation & 15,058 & 439 & 2.9 & 42.4 & 161 & 36.7 & 38.9 \\
\hline Bowel function & 16,303 & 678 & 4.2 & 37.5 & 325 & 47.9 & 35.5 \\
\hline Hormone function & 16,945 & 1669 & 9.9 & 37.2 & 821 & 49.2 & 35.3 \\
\hline Sexual function & 17,386 & 5968 & 34.3 & 2.8 & 4243 & 71.1 & 2.3 \\
\hline
\end{tabular}

*The threshold for poor function is a score $<50$ for urinary incontinence, urinary irritation, bowel function and hormone function and a score of $<10$ for sexual function

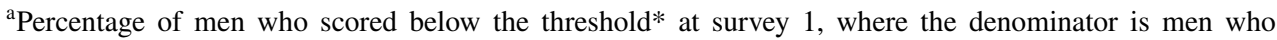
reported no additional treatment at follow-up

${ }^{b}$ Percentage of men who continued to score below the threshold* at survey 2, where the denominator is men who scored below the threshold at survey 1 treatment involving EBRT, brachytherapy or ADT (either alone, combined or with systemic treatment) (Table 2).

\section{Sexual function}

A marked decline in sexual function was reported by all groups who reported their first active treatment at follow-up (Table 2). Mean sexual function scores in men who reported moving to EBRT (alone or combined with ADT) or brachytherapy were 17.9 points lower at follow-up (decreasing from 46.5 to 28.6). In men whose first active treatment was surgery, scores were on average 24.9 points lower at followup (decreasing from 50.5 to 25.6).

\section{Generic HRQL}

Patients who had ADT, EBRT or brachytherapy as their first active treatment reported more problems with all EQ-5D dimensions at follow-up. For example, $75.1 \%$ of ADT patients reported $\geq 1$ problem at follow-up (a 7.9\% increase) and $67.7 \%$ of EBRT or brachytherapy patients reported $\geq 1$ problem at follow-up (a $12.3 \%$ increase). These issues do not appear to impact on SAH, with no clinically meaningful changes in scores observed (Table 4).

\section{Respondents who reported additional active treatment at follow-up}

\section{Urinary and bowel function}

CMDs (declines) in urinary incontinence scores were observed for men who reported EBRT and ADT in the first survey and either surgical or systemic treatments at followup ( -6.0 points and -6.7 points, respectively), although these groups were relatively small (Table 2). Men who reported the addition of EBRT reported worse bowel function at follow-up, with a CMDs for men treated with EBRT following initial ADT ( -5.8 points) and men treated with combined EBRT and ADT following surgery ( -6.7 points) (Table 2).

\section{Vitality and Hormone function}

Men who reported additional combined EBRT and ADT at follow-up, having previously reported only surgical treatment, reported a clinically meaningful 5 point decline in hormone function (Table 2). Men who reported a change in treatment from combined EBRT and ADT (likely ceasing treatment) to surgical treatment report an improvement in hormone function ( +4 points, from 67.6 to 71.6 ). 


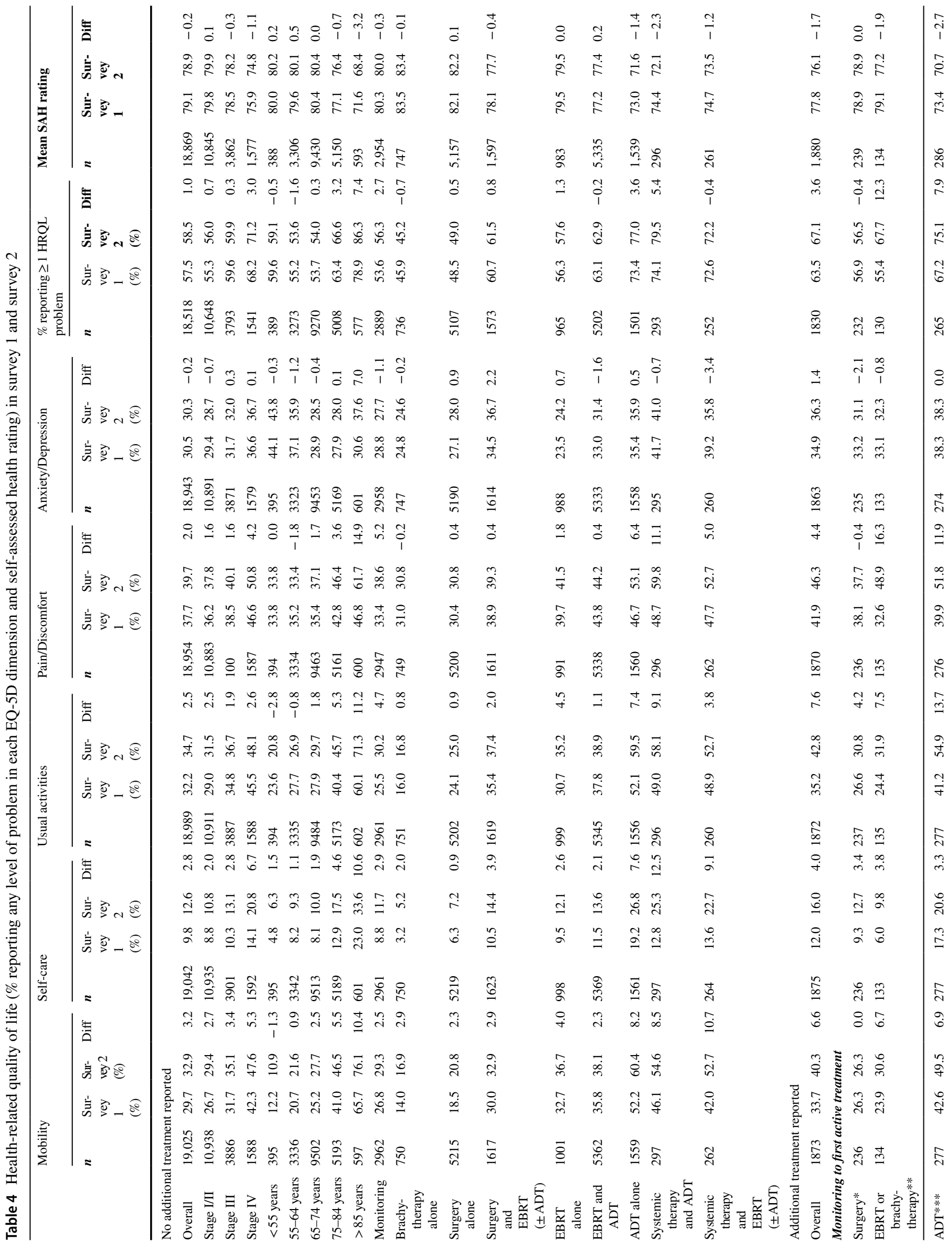




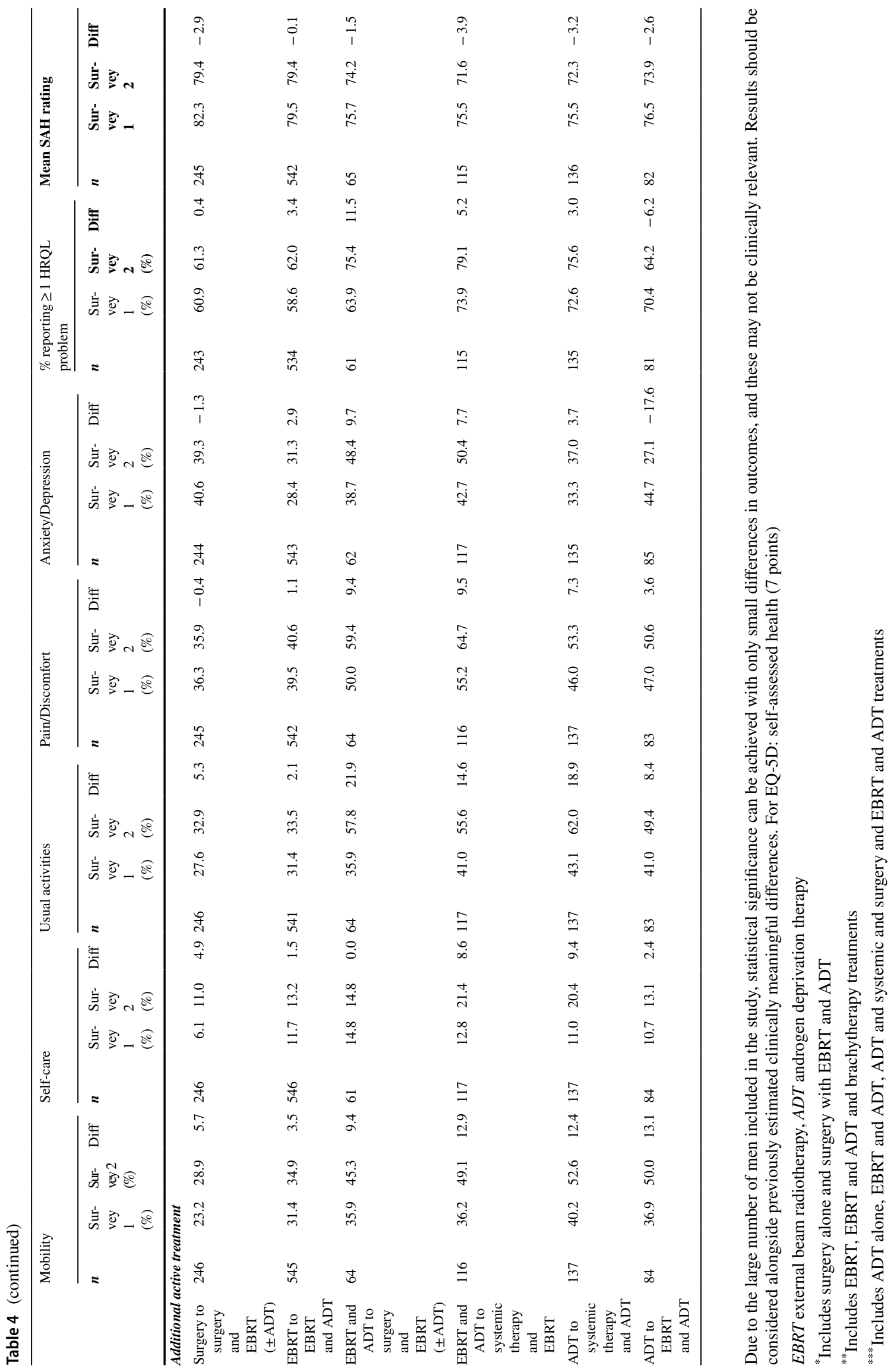




\section{Sexual function}

Mean scores for sexual function declined between surveys in all treatment groups, but the differences observed were not clinically meaningful (Table 2 ).

\section{Generic HRQL}

Overall, men who received additional treatment between surveys reported more HRQL problems at follow-up (across all EQ-5D dimensions and SAH) (Table 4). The lowest SAH ratings and largest reductions in SAH were observed in men moving to systemic treatments $(-3.2$ point change for men reporting ADT and systemic therapy at follow-up and -3.9 point change for men reporting EBRT and systemic therapy at follow-up).

\section{Discussion}

We report on a follow-up of the largest PCa patientreported outcome study in the world to date, evaluating the on-going HRQL of the LAPCD cohort. Evidence from this study suggests stability of HRQL and most specific morbidities by $18-42$ months for men who report no further active treatment in the subsequent 12 months, including those with advanced disease. However, this includes men who reported poor function in the original survey and continued to report poor function. At follow-up, $10 \%$ of the cohort reported receiving additional treatment or their first active treatment, which for many will be as a result of disease recurrence or progression.

The largest improvements in function were observed for hormonal issues, such as weight change, hot flushes, fatigue and depression. These are well-known side effects of ADT and were shown to be a major problem for men in the first LAPCD survey [14]. A clinically meaningful improvement in hormone function was observed in men who reported no further treatment following combined EBRT and ADT. It is documented that some men find their side effects improve or become more manageable the longer they are on ADT, while others find that side effects improve once they have stopped therapy and testosterone levels rise, although this can take several months or years [26]. It is therefore plausible that improvements were reported by this cohort because they either stopped or became accustomed to the impact of ADT. Unfortunately, we do not have data relating to the length of time that men were on ADT.

Although PCa-specific outcomes were stable in the year following the first survey, this includes continued poor sexual function across all treatment and sociodemographic cohorts. When looking at men who had completed both surveys and who reported no additional treatment at follow-up, few reported an improvement in function 12 months later. The lack of access to interventions to aid sexual function has been highlighted through the LAPCD study [14].

Another group requiring continued support is men experiencing poor urinary function, which is common following surgery. Almost three-quarters of surgical patients who reported poor continence in the first survey did not improve over the next year. Longitudinal research has shown that by 48 months, post-diagnosis urinary incontinence scores were significantly worse in surgical patients compared to other patient cohorts [27]. A study which followed patients for 15 years found that while urinary incontinence was less prevalent than sexual dysfunction, it was a greater cause of bother [11]. Support can be offered in a variety of ways, and possibilities for improving continence exist, including bladder retraining, pelvic floor exercises and medical interventions [28]. Clinicians should be encouraged to ask about urinary function in follow-up clinics, and men should be informed of the risk of longerterm quality of life issues.

Our results additionally identify a requirement to continue to support men undergoing ADT treatment. Previous research based on the LAPCD cohort has shown that worse cancer-specific and generic HRQL is associated with psychological distress and poor mental wellbeing in men treated with ADT [29]. This further emphasises the wider impact of cancer-specific HRQL. To date, interventions with ADT patients focus on lifestyle changes to reduce side effects and risk of developing further comorbidities from treatment [30].

Despite continued issues with urinary, bowel and sexual issues, overall, there was little change in HRQL among any of the treatment cohorts. These findings lend more support to the idea of the 'gap hypothesis' or 'response shift' of HRQL, the theory that being diagnosed with a life-threatening illness may result in patients re-evaluating what is important to them and re-calibrating expectations of what life with cancer will be like [31,32].

Our results indicated that, of the 588 men who reported being on monitoring at initial survey and active treatment at follow-up, 39.8\% reported subsequent surgery (alone) and $29.3 \%$ reported moving to ADT (alone). When compared to figures reported by PROTECT, which reported on men diagnosed with early stage disease only, our results are consistent for surgery, where half of the PROTECT cohort who started on monitoring moved to surgical treatment [10]. However, our results showed that only $5.6 \%$ of men were treated with radiotherapy alone after monitoring, which differ substantially from those reported by PROTECT, where a third of the cohort moved to radiotherapy after monitoring. These differences are not unexpected due to the LAPCD study including 
patients with both early stage and advanced disease, with many men receiving combined EBRT and ADT.

Treatment information was self-reported at both the initial and follow-up surveys. As such, there were some difficulties in trying to categorise responses into either 'no further treatment' or 'additional treatment'. For example, many men reported an active treatment in the original survey but then reported active surveillance or watchful waiting at follow-up, which we interpreted as clinical monitoring and therefore no further active treatment. There were also instances where we believe that men reported only their current treatment in the follow-up survey rather than all received treatments. Due to these difficulties, some respondents had to be excluded from analyses.

There were some limitations in data interpretation. The first was that, due to privacy restrictions, we were unable to access date of diagnosis. This meant that outcomes could not be stratified by time since diagnosis, as information was not available as to how far post-diagnosis men were. The men included in this study are therefore a heterogenous group of medium- to long-term survivors. The second was that we did not have information about disease progression in the time since the first survey, as such data is not currently captured accurately by cancer registries. Finally, we were not definitively able to identify which men had finished treatment, which men were still receiving treatment and when they had last been treated. These factors will have some impact on HRQL outcomes but could not be investigated fully in this current study. Future research would benefit from recording this information as it may assist in providing greater understanding why some men experience worse or continued poor HRQL.

\section{Conclusions}

Overall, patient-reported outcomes in men with $\mathrm{PCa}$ remained generally stable, which is reassuring for those with good function and HRQL but re-enforces the need for early intervention and support for those who experience poor outcomes, as these seem to persist for the majority. There remains a specific need to provide on-going support to men who have undergone ADT or surgical treatments as a high proportion of them report persistent problems. Poorer HRQL and specific functional problems were reported by men who received additional treatment and men who received their first active treatment between the two surveys: essentially, these patients start back at the beginning on their HRQL journey. These results further highlight that men living with and beyond $\mathrm{PCa}$ require patient-centred services to address treatment side effects, with the goal of enhancing their HRQL.
Supplementary Information The online version contains supplementary material available at https://doi.org/10.1007/s00520-021-06650-7.

Acknowledgements We thank all the men who completed surveys. We acknowledge the following people and groups for their contribution to the development and running of the study: Rebecca Mottram, Majorie Allen, Therese Kearney, Oonagh McSorley, Heather Kinnear, Victoria Cairnduff, Linda Roberts, Conan Donnelly, Emma McNair, Fraser Munro, Dawn Allen, Janet Warlow, the User Advisory Group, the Clinical and Scientific Advisory Group and Picker Institute Europe (Oxford, UK). This study is based in part on information collected and quality assured by the cancer registries in each nation. Their work uses data provided by patients and collected by health services as part of their care and support.

Author contribution AG and AWG are co-principal Investigators and designed the study together with co-investigators AD, PW, LH, EW, RW, PS, PK and HB. SM and AD analysed the data. SW, PW, LH, EW, RW and PS advised on interpretation of the data. HB chaired the User Advisory Group and provided input from a patient perspective. PS chaired the Clinical \& Scientific Advisory Group. SM wrote the initial draft of the paper. All authors contributed to critically revising the paper and approved the final version.

Funding The Life After Prostate Cancer Diagnosis study was funded by the Movember Foundation, in partnership with Prostate Cancer UK, as part of the Prostate Cancer Outcomes programme, grant number BO26/MO.

Data availability The individual patient-level data used to generate results are not freely available but may be applied for through the Public Health England Office for Data Release.

Code availability Stata code is available from the authors upon request.

\section{Declarations}

Ethics approval The study was approved by the following organisations: Newcastle and North Tyneside 1 Research Ethics Committee (15/ NE/0036), Confidentiality Advisory Group (15/CAG/0110), NHS Scotland Public Benefit and Privacy Panel (0516-0364) and NHS Research and Development approval from Wales, Scotland and Northern Ireland.

Consent to participate Permission to approach patients without informed consent was given by the Confidentiality Advisory Group (15/CAG/0110). Men consented to the study by completing and returning the surveys.

Consent for publication By completing and returning surveys, men consented to their data being used in future publications. All data is pseudonymised, and no men can be identified from the aggregated results.

Conflict of interest The authors declare no competing interests.

Open Access This article is licensed under a Creative Commons Attribution 4.0 International License, which permits use, sharing, adaptation, distribution and reproduction in any medium or format, as long as you give appropriate credit to the original author(s) and the source, provide a link to the Creative Commons licence, and indicate if changes were made. The images or other third party material in this article are included in the article's Creative Commons licence, unless indicated otherwise in a credit line to the material. If material is not included in 
the article's Creative Commons licence and your intended use is not permitted by statutory regulation or exceeds the permitted use, you will need to obtain permission directly from the copyright holder. To view a copy of this licence, visit http://creativecommons.org/licenses/by/4.0/.

\section{References}

1. Office for National Statistics. Cancer survival in England: national estimates for patients followed up to 2017. Accessed: 11/02/2020. Available from: https://www.ons.gov.uk/peoplepopulationandc ommunity/healthandsocialcare/conditionsanddiseases/bulletins/ cancersurvivalinengland/nationalestimatesforpatientsfollowe dupto2017.

2. Glaser AW, Corner JL (2015) Prostate cancer outcomes: the three questions. Eur Urol 67(3):357-358

3. Huang GJ, Sadetsky N, Penson DF (2010) Health related quality of life for men treated for localized prostate cancer with long-term followup. J Urol 183(6):2206-2212

4. Pardo Y, Guedea F, Aguilo F, Fernandez P, Macias V, Marino A et al (2010) Quality-of-life impact of primary treatments for localized prostate cancer in patients without hormonal treatment. J Clin Oncol 28(31):4687-4696

5. Resnick MJ, Koyama T, Fan K-H, Albertsen PC, Goodman M, Hamilton AS et al (2013) Long-term functional outcomes after treatment for localized prostate cancer. N Engl J Med 368(5):436-445

6. Sanda MG, Dunn RL, Michalski J, Sandler HM, Northouse L, Hembroff L et al (2008) Quality of life and satisfaction with outcome among prostate-cancer survivors. N Engl J Med 358(12):1250-1261

7. Punnen S, Cowan JE, Chan JM, Carroll PR, Cooperberg MR (2015) Long-term health-related quality of life after primary treatment for localized prostate cancer: results from the CaPSURE registry. Eur Urol 68(4):600-608

8. Shahinian VB, Kuo YF, Freeman JL, Goodwin JS (2006) Risk of the "androgen deprivation syndrome" in men receiving androgen deprivation for prostate cancer. Arch Intern Med 166(4):465-471

9. Alibhai SM, Breunis H, Timilshina N, Johnston C, Tomlinson G, Tannock I et al (2010) Impact of androgen-deprivation therapy on physical function and quality of life in men with nonmetastatic prostate cancer. J Clin Oncol 28(34):5038-5045

10. Hamdy FC, Donovan JL, Lane JA, Mason M, Metcalfe C, Holding $\mathrm{P}$ et al (2016) 10-year outcomes after monitoring, surgery, or radiotherapy for localized prostate cancer. N Engl J Med 375(15):1415-1424

11. Mazariego CG, Egger S, King MT, Juraskova I, Woo H, Berry M, et al. Fifteen year quality of life outcomes in men with localised prostate cancer: population based Australian prospective study. BMJ. 2020;371:m3503.

12. Downing A, Wright $\mathrm{P}$, Wagland R, Watson E, Kearney T, Mottram R, et al. Life after prostate cancer diagnosis: protocol for a UK-wide patient-reported outcomes study. BMJ Open. 2016;6(12):e013555.

13. Downing A, Morris EJ, Richards M, Corner J, Wright P, SebagMontefiore D et al (2015) Health-related quality of life after colorectal cancer in England: a patient-reported outcomes study of individuals 12 to 36 months after diagnosis. J Clin Oncol 33(6):616-624

14. Downing A, Wright P, Hounsome L, Selby P, Wilding S, Watson E et al (2019) Quality of life in men living with advanced and localised prostate cancer in the UK: a population-based study. Lancet Oncol 20(3):436-447
15. Williams A (1990) EuroQol-a new facility for the measurement of health-related quality of life. Health Policy 16(3):199-208

16. Herdman M, Gudex C, Lloyd A, Janssen M, Kind P, Parkin D et al (2011) Development and preliminary testing of the new five-level version of EQ-5D (EQ-5D-5L). Qual Life Res 20(10):1727-1736

17. Wei JT, Dunn RL, Litwin MS, Sandler HM, Sanda MG (2000) Development and validation of the expanded prostate cancer index composite (EPIC) for comprehensive assessment of health-related quality of life in men with prostate cancer. Urology 56(6):899-905

18. University of Michigan. Scoring instructions for the Expanded Prostate Cancer Index Composite short form (EPIC-26). 2002. Accessed 11/02/2020. Available from: https://medicine.umich. edu/sites/default/files/content/downloads/Scoring\%20Instruct ions\%20for\%20the\%20EPIC\%2026.pdf.

19. Department for Communities and Local Government. English indices of multiple deprivation 2010. Accessed 11/02/2020. Available from: https://www.gov.uk/government/statistics/english-indic es-of-deprivation-2010.

20. ISD Scotland. The Scottish Index of Multiple Deprivation (SIMD) 2017. Accessed 11/02/2020. Available from: http://www.isdsc otland.org/Products-and-Services/GPD-Support/Deprivation/ SIMD/.

21. Northern Ireland Statistics and Research Agency. NI Multiple Deprivation Measure 2010. Accessed 11/02/2020. Available from: https://www.nisra.gov.uk/statistics/deprivation/northern-irelandmultiple-deprivation-measure-2010-nimdm2010.

22. Welsh Government. Welsh Index of Multiple Deprivation 2017. Accessed 11/02/2020. Available from: http://wimd.wales.gov.uk/? lang=en.

23. Pickard AS, Neary MP, Cella D (2007) Estimation of minimally important differences in EQ-5D utility and VAS scores in cancer. Health Qual Life Outcomes 5(1):70

24. Watson E, Shinkins B, Frith E, Neal D, Hamdy F, Walter F et al (2016) Symptoms, unmet needs, psychological well-being and health status in survivors of prostate cancer: implications for redesigning follow-up. BJU Int 117(6b):E10-E19

25. Skolarus TA, Dunn RL, Sanda MG, Chang P, Greenfield TK, Litwin MS et al (2015) Minimally important difference for the Expanded Prostate Cancer Index Composite Short Form. Urology 85(1):101-105

26. Prostate Cancer UK. Hormone therapy. Accessed 11/02/2020. Available from: https://prostatecanceruk.org/prostate-information/ treatments/hormone-therapy.

27. Zelefsky MJ, Poon BY, Eastham J, Vickers A, Pei X, Scardino PT (2016) Longitudinal assessment of quality of life after surgery, conformal brachytherapy, and intensity-modulated radiation therapy for prostate cancer. Radiother Oncol 118(1):85-91

28. Prostate Cancer UK. Treatments for leaking urine. Accessed 11/02/2020. Available from: https://prostatecanceruk.org/prost ate-information/guides/how-to-manage/urinary-problems/leaki ng-urine/treatments.

29. Wilding S, Downing A, Wright P, Selby P, Watson E, Wagland R et al (2019) Cancer-related symptoms, mental well-being, and psychological distress in men diagnosed with prostate cancer treated with androgen deprivation therapy. Qual Life Res 28:2741-2751

30. Ying M, Zhao R, Jiang D, Gu S, Li M (2018) Lifestyle interventions to alleviate side effects on prostate cancer patients receiving androgen deprivation therapy: a meta-analysis. Jpn J Clin Oncol 48(9):827-834

31. Calman KC (1984) Quality of life in cancer patients-an hypothesis. J Med Ethics 10(3):124-127

32. Schwartz CE, Andresen EM, Nosek MA, Krahn GL (2007) Response shift theory: important implications for measuring quality of life in people with disability. Arch Phys Med Rehabil 88(4):529-536 
Publisher's Note Springer Nature remains neutral with regard to jurisdictional claims in published maps and institutional affiliations. 